\title{
Independent Inhibition of the Polymerase and Deubiquitinase Activities of the Crimean-Congo Hemorrhagic Fever Virus Full-Length L-Protein ${ }^{\dagger}$
}

\author{
Egor P. Tchesnokov ${ }^{1}$, Ben A. Bailey-Elkin ${ }^{2}$, Brian L. Mark ${ }^{2}$ and Matthias Götte ${ }^{1,2, *}$ \\ 1 Department of Medical Microbiology and Immunology, Li Ka Shing Institute of Virology, \\ University of Alberta, Edmonton, AB T6G 2E1, Canada; tchesnok@ualberta.ca \\ 2 Department of Microbiology, University of Manitoba, Winnipeg, MB R3T 2N2, Canada; \\ Ben.Bailey-Elkin@umanitoba.ca (B.A.B.-E.); brian.mark@umanitoba.ca (B.L.M.) \\ * Correspondence: gotte@ualberta.ca \\ † Presented at Viruses 2020-Novel Concepts in Virology, Barcelona, Spain, 5-7 February 2020. \\ Published: 13 June 2020
}

\begin{abstract}
The Crimean-Congo hemorrhagic fever virus (CCHFV) is a segmented negative-sense RNA virus that can cause severe human disease. The World Health Organization (WHO) has listed CCHFV as a priority pathogen with an urgent need for enhanced research activities to develop effective countermeasures. We report on the expression, characterization, and inhibition of the CCHFV full-length L-protein that provides an important tool in this regard. The requirements for high biosafety measures hamper drug discovery and development efforts with infectious CCHFV. Hence, we decided to adopt a biochemical approach that targets the viral RNA-dependent RNA polymerase (RdRp). The CCHFV RdRp activity is part of a multifunctional L protein that is unusually large, with a molecular weight of $\sim 450 \mathrm{kDa}$. The CCHFV L-protein also contains an ovarian tumor (OTU) domain that exhibits deubiquitinating (DUB) activity. Previous studies have shown that DUB activity interferes with innate immune responses and viral replication. Here, we utilized the baculovirus expression system and generated a full-length CCHFV L protein. RdRp activity was seen in the presence of divalent metal ions, and inhibition of RNA synthesis was demonstrated with nucleotide analogues. The ubiquitin analogue CC.4 inhibits the CCHFV-associated DUB activity of the full-length L protein and the isolated DUB domain to a similar extent. We have finally shown that RdRp and DUB activities are functionally independent. The full-length CCHFV L-protein provides an important tool for the discovery of antiviral agents. High-throughput screening (HTS) campaigns are now feasible. The same enzyme preparations can be utilized to identify polymerase and DUB inhibitors.
\end{abstract}

Keywords: RNA-dependent RNA polymerase; deubiquitinase; viral replication

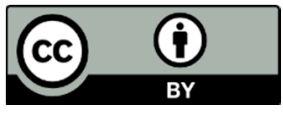

(C) 2020 by the authors. Licensee MDPI, Basel, Switzerland. This article is an open access article distributed under the terms and conditions of the Creative Commons Attribution (CC BY) license (http://creativecommons.org/licenses/by/4.0/). 